\title{
Aortic arch interruption
}

INSERM

\section{Source}

INSERM. (1999). Orphanet: an online rare disease and orphan drug data base. Aortic arch interruption. ORPHA:2299

Aortic arch interruption is a rare heart defect characterized by complete lack of anatomical continuity between the transverse aortic arch and the descending thoracic aorta. AAl should be distinguished anatomically from atresia of the aortic arch where continuity between these segments is achieved by an imperforate fibrous strand of various lengths. 